\title{
The orbital stability of the cnoidal waves of the Korteweg-de Vries equation
}

\author{
Bernard Deconinck * \\ Department of Applied Mathematics \\ University of Washington \\ Seattle, WA 98195
}

\author{
Todd Kapitula ${ }^{\dagger}$ \\ Department of Mathematics and Statistics \\ Calvin College \\ Grand Rapids, MI 49546
}

April 8, 2010

\begin{abstract}
The cnoidal wave solution of the integrable Korteweg - de Vries equation is the most basic of its periodic solutions. Following earlier work where the linear stability of these solutions was established, we prove in this paper that cnoidal waves are (nonlinearly) orbitally stable with respect to so-called subharmonic perturbations: perturbations that are periodic with period any integer multiple of the cnoidal-wave period. Our method of proof combines the construction of an appropriate Lyapunov function with the seminal results of Grillakis, Shatah and Strauss $[\mathbf{1 4}, \mathbf{1 5}]$. The integrability of the Korteweg-de Vries equation is used in that we need the presence of at least one extra conserved quantity in addition to those expected from the Lie point symmetries of the equation.
\end{abstract}

\section{INTRODUCTION}

The stability of spatially periodic stationary solutions of nonlinear wave equations has seen different advances the past few years. There are advances both in the numerical investigation of spectral stability $[\mathbf{1 0}, \mathbf{2 2}]$, as well as in the analytical study of spectral and orbital stability (see $[\mathbf{3}, \mathbf{6}, \mathbf{7}, \mathbf{1 1}, \mathbf{1 2}, \mathbf{1 6}]$ and the references therein). For integrable equations such as the Korteweg-de Vries (KdV) equation,

$$
u_{t}+\left(\frac{u^{2}}{2}+u_{x x}\right)_{x}=0
$$

the investigations can be made more concrete, leading to an explicit determination of the spectrum of the operator for the spectral stability problem. We show in this paper that explicit orbital stability results can also be obtained.

We consider the cnoidal wave solutions of (1.1):

$$
u(x, t)=12 k^{2} \operatorname{cn}^{2}(x-c t, k), \quad c=8 k^{2}-4 .
$$

Here $\operatorname{cn}(x, k)$ is the Jacobi elliptic cosine function with elliptic modulus $k \in[0,1)[\mathbf{2}, \mathbf{8}]$. It generalizes the cosine function to which it is equal for $k=0$, and limits to a hyperbolic secant as $k \rightarrow 1^{-}$. The cnoidal wave is periodic with period $T(k)=2 K(k)$, where $K(k)$ is the complete elliptic integral of the first kind $[\mathbf{2}, \mathbf{8}]$ given by

$$
K(k)=\int_{0}^{\pi / 2} \frac{1}{\sqrt{1-k^{2} \sin ^{2} z}} \mathrm{~d} z .
$$

\footnotetext{
*E-mail: bernard@amath.washington.edu

†E-mail: tmk5@calvin.edu
} 
The cnoidal waves are the simplest of the periodic solutions of the KdV equation, represent the most general stationary (in a frame translating with speed $c$ ) solution of the equation, and contain the famous soliton solution as a limit case. The solutions are of practical importance in many applications such as, for instance, near-shore ocean waves [23]. As such the issue of their stability is important. Further, it is clear from this particular application that it is insufficient to consider the stability of the cnoidal waves with respect to perturbations of period $T(k)$. Indeed, physically the only restriction on the perturbations is that they be continuous and bounded on $x \in \mathbb{R}$. In order to work in a more friendly setting in terms of the function space we can consider, we restrict ourselves to so-called subharmonic perturbations ${ }^{1}$ : perturbations that are periodic with period $n T(k)$, where $n$ is any nonzero positive integer. Note that the class of subharmonic perturbations is larger than that of perturbations of the same period, and is a significant step closer to the desired class of perturbations.

The spectral and linear stability of the cnoidal waves were the subject of [6], and their orbital stability with respect to harmonic (same period) perturbations was covered in [21] (using integrability) and [3] (not using integrability). Leaving the realm of harmonic perturbations leaves us with the question posed by Benjamin in 1972 [4, Page 12]:

"The question thus arises whether, when released for the present sufficient condition for stability (i.e. restriction to perturbations with the same period), periodic waves become unstable."

In other words, Benjamin was asking whether waves which are energetically unstable, but are spectrally stable, can be orbitally stable. Benjamin was exploring the possibility of extending the methods he used to establish the orbital stability of the soliton solution of the KdV equation to the stability analysis of the cnoidal wave solution. His considerations indicated that such should be possible when considering harmonic perturbations. In that case it appeared a Lyapunov function could be used which was in essence the energy function for the KdV equation, see below. This indication was proven to be correct more than 30 years later [3]. Next, Benjamin showed that these methods would fail, and the energy functional would not act as a Lyapunov functional for the cnoidal waves, when considering subharmonic perturbations (even when their period was only twice the period of the cnoidal wave). He examined this problem in some detail and provided some indications that point to the instability of the cnoidal waves with respect to this class of perturbations. Ultimately, no rigorous results were found, and the problem remained open. Bottman and Deconinck [6] were able to confirm the spectral and linear stability of the cnoidal waves with respect to bounded perturbations.

Up until now the question of the orbital stability of these waves with respect to subharmonic perturbations was open, primarily because in this case the wave is not a local minimizer of a constrained energy. By using the integrable structure associated with $\mathrm{KdV}$, the results of $[\mathbf{6}]$ on the nature of the spectrum for the linearized operator, and the Lyapunov function construction ideas presented in [20], we are able to show that these energetically unstable waves are indeed orbitally stable.

Acknowledgments. BD acknowledges support from the National Science Foundation through grant NSFDMS-0604546. TK gratefully acknowledges the support of the Jack and Lois Kuipers Applied Mathematics Endowment, a Calvin Research Fellowship, and the National Science Foundation under grant DMS-0806636. Any opinions, findings, and conclusions or recommendations expressed in this material are those of the authors and do not necessarily reflect the views of the funding sources.

\section{LiNEAR STABILITY RESUlTS FOR THE CNOIDAL WAVE SOLUTIONS}

We rewrite the $\mathrm{KdV}$ equation in a frame moving with constant velocity $c$, so that the cnoidal wave is a stationary (i.e., time-independent) solution of the equation. Let

$$
y=x-c t, \quad \tau=t
$$

The KdV equation becomes

\footnotetext{
${ }^{1}$ Note that superharmonic perturbations (perturbations whose period is the base period divided by a positive $(>1)$ integer) are covered by studying perturbations that have the same period as the underlying solution.
} 


$$
u_{\tau}+\left(\frac{u^{2}}{2}+u_{y y}-c u\right)_{y}=0
$$

with stationary solution

$$
U(y)=12 k^{2} \operatorname{cn}^{2}(y, k) .
$$

The linear stability problem for this solution is given by

$$
v_{\tau}+\left(U v+v_{y y}-c v\right)_{y}=0
$$

obtained by discarding terms of order two or higher in (2.2), when we let $u(y, \tau)=U(y)+\epsilon v(y, \tau)+\mathcal{O}\left(\epsilon^{2}\right)$. The study of this linear stability problem is the focus of [6]. In this section, we review those results from [6] that are necessary for our considerations below.

The linear stability problem (2.4) is autonomous in $\tau$. A separation of variables $v(y, \tau)=e^{\lambda \tau} V(y)$ yields the spectral problem

$$
\lambda V=\partial_{y} \mathcal{L} V, \quad \mathcal{L}=-\partial_{y}^{2}+c-U
$$

Spectral stability of the cnoidal wave (2.3) with respect to perturbations that are bounded on the whole line is established by demonstrating that the spectrum $\sigma\left(\partial_{y} \mathcal{L}\right)$ of the operator $\partial_{y} \mathcal{L}$ does not intersect the right-half complex $\lambda$ plane. Since (2.2) is a Hamiltonian partial differential equation [13], the spectrum $\sigma\left(\partial_{y} \mathcal{L}\right)$ is symmetric under reflection with respect to both the real and imaginary axes. As a consequence, spectral stability requires proving that $\sigma\left(\partial_{y} \mathcal{L}\right)$ is confined to the imaginary axis. In order to do so, Bottman \& Deconinck [6] exploit the squared-eigenfunction connection [1] between solutions of (2.4) and the components of the eigenfunction of the Lax pair for the KdV equation. We review the steps of this process.

The KdV equation $(2.2)$ is an integrable equation $[\mathbf{6}, \mathbf{1 3}, \mathbf{1 9}]$ with Lax pair given by

$$
\psi_{y}=\left(\begin{array}{cc}
0 & 1 \\
\zeta-u / 6 & 0
\end{array}\right) \psi
$$

and

$$
\psi_{\tau}=\left(\begin{array}{cc}
u_{y} / 6 & -4 \zeta+c-u / 3 \\
-4 \zeta^{2}+c \zeta+\left(u^{2}-3 c u+6 \zeta u+3 u_{y y}\right) / 18 & -u_{y} / 6
\end{array}\right) \psi
$$

Since (2.6) is equivalent to the linear Schrödinger equation, the spectral parameter is contained in a subset of the real line. By restricting the Lax pair (2.6-2.7) to the stationary solution $u(y, \tau)=U(y)$, the spectrum $\sigma_{L}$ of the Lax problem (2.6) can be determined explicitly. First, (2.7) with $u(y, \tau)=U(y)$ is autonomous in $\tau$, from which it follows that

$$
\psi(y, \tau)=e^{\Omega \tau}\left(\begin{array}{c}
\alpha(y) \\
\beta(y)
\end{array}\right) .
$$

A priori, the separation constant $\Omega$ may depend on both $y$ and $\zeta$. Using the commutativity of (2.6) and (2.7), it follows that $\Omega$ does not depend on $y$; in fact, one finds explicitly

$$
\Omega^{2}=16\left(\zeta-k^{2}+1\right)\left(\zeta-2 k^{2}+1\right)\left(\zeta-k^{2}\right)
$$

The vector $(\alpha, \beta)^{T}$ is easily determined from (2.7) and subsequent use of (2.6). One finds

$$
\left(\begin{array}{c}
\alpha(y) \\
\beta(y)
\end{array}\right)=\gamma_{0} \exp \left(\int \frac{-U_{y} / 6-\Omega}{4 \zeta-c+U / 3} \mathrm{~d} y\right)\left(\begin{array}{c}
4 \zeta-c+U / 3 \\
U_{y} / 6-\Omega
\end{array}\right)
$$

where $\gamma_{0}$ is a real constant. Using the explicit form of the eigenfunction $\psi$, the statement $\zeta \in \sigma_{L}$ is found to be equivalent with

$$
\operatorname{Re}\left\langle\frac{-U_{y} / 6-\Omega}{4 \zeta-c+U / 3}\right\rangle=0
$$


where $\langle\cdot\rangle$ denotes the spatial average of its argument. From this, it follows easily that

$$
\sigma_{L}=\left\{\zeta \in \mathbb{R}: \zeta \in\left(-\infty, k^{2}-1\right] \cup\left[2 k^{2}-1, k^{2}\right]\right\} .
$$

Having determined the solution of the Lax pair equations (2.6-2.7), we turn to the squared eigenfunction connection, from which it follows that $\psi_{1}(y, \tau) \psi_{2}(y, \tau)$ solves $(2.4)$, see [6]. The conclusion that $\alpha(y) \beta(y)$ solves (2.5) with $\lambda=2 \Omega$ is immediate. In fact, it is shown in [6] that all bounded solutions of (2.5) are obtained this way. Since for the values of $\zeta \in \sigma_{L}, \lambda=2 \Omega$ is imaginary, the spectral stability of the cnoidal wave solutions follows. An application of the SCS Basis Lemma [16] allows for the conclusion of linear stability.

\section{ORBital STABILITY OF THE CNOIDAL WAVE SOLUTIONS}

Using the results reviewed in the previous section, we now show that the cnoidal waves (2.3) are stable when considered on the space $L_{\text {per }}^{2}([-n K(k), n K(k)])$ for any $n \in \mathbb{N}$, i.e., with respect to subharmonic perturbations. In essence our approach follows the algorithm outlined in [17]. Using a sufficiently large number of conserved quantities of the KdV equation, we are able to construct a Lyapunov functional for the cnoidal waves, hence establishing so-called formal stability. Next, we verify that the additional assumptions of Theorem 1 in [14] are satisfied, from which orbital stability follows.

We first show that the cnoidal waves are not constrained minimizers of the "natural" energy for the KdV equation. This problem is overcome by considering the extra conserved quantities of the KdV equation, as shown explicitly below. The fact that we are able to construct a Lyapunov functional when it is clear the energy cannot play this role, is a consequence of the integrability of the $\mathrm{KdV}$ equation, which provides us with an infinite number of candidate Lyapunov functionals. This same idea was used recently to establish the orbital stability of the multi-soliton solutions of equations in the AKNS hierarchy [18]. Particularly relevant for us is the seminal work of [20], as it is particularly devoted to the case of the KdV equation.

Note that the operator $\mathcal{L}$ is the Hessian (second variation) of the Hamiltonian

$$
H=\frac{1}{2} \int_{-n K(k)}^{n K(k)}\left(u_{y}^{2}+c u^{2}-\frac{1}{3} u^{3}\right) \mathrm{d} y
$$

for the KdV equation (2.2), evaluated at the cnoidal wave. We begin by calculating the Krein signature associated with the operator $\mathcal{L}$, parametrized by the Lax spectral parameter $\zeta$. The Krein signature is defined as the sign of

$$
\mathcal{K}_{1}=\langle V, \mathcal{L} V\rangle_{n}:=\int_{-n K(k)}^{n K(k)} V^{*} \mathcal{L} V \mathrm{~d} y
$$

where $n \in \mathbb{N}$ and $V$ is a solution of the spectral problem (2.5). We wish to calculate $\mathcal{K}_{1}$ for all bounded $V$. As was shown in [6] and stated in the previous section, all such $V$ are of the form $V=\alpha \beta$ for all $\zeta \in \sigma_{L}$, and we may consider $\mathcal{K}_{1}$ as being parameterized by the Lax spectral parameter $\zeta \in \sigma_{L}$. We have

$$
V=\gamma^{2}\left(4 \zeta-c+\frac{U}{3}\right)\left(\frac{U_{y}}{6}-\Omega(\zeta)\right) .
$$

for $\zeta \in\left(-\infty, k^{2}-1\right] \cup\left[2 k^{2}-1, k^{2}\right]$. Further,

$$
\gamma(y)=\gamma_{0} \exp \left(\int \frac{-U^{\prime} / 6-\Omega(\zeta)}{4 \zeta-c+U / 3} \mathrm{~d} y\right)=\frac{\gamma_{0}}{2\left(\zeta-k^{2}-k^{2} \operatorname{sn}^{2}(y, k)+1\right)^{1 / 2}} \mathrm{e}^{\mathrm{i} \arg \gamma(y)},
$$

where $\arg \gamma(x)$ is the argument of $\gamma(x)$. Note that (3.3) represents two eigenfunctions, one for each choice of the sign of $\Omega(\zeta)$.

A direct calculation shows that the integrand of $\mathcal{K}_{1}(\zeta)$ is given by 


$$
\begin{aligned}
V^{*} \mathcal{L} V & =-64|\gamma(y)|^{4} \Omega(\zeta)\left(\zeta-k^{2}-k^{2} \operatorname{sn}^{2}(y, k)+1\right)^{3}\left(4 k^{2} \operatorname{cn}(y, k) \operatorname{dn}(y, k) \operatorname{sn}(y, k)-\Omega(\zeta)\right) \\
& =-4 \Omega(\zeta)\left(\zeta-k^{2}-k^{2} \operatorname{sn}^{2}(y, k)+1\right)\left(4 k^{2} \operatorname{cn}(y, k) \operatorname{dn}(y, k) \operatorname{sn}(y, k)-\Omega(\zeta)\right)
\end{aligned}
$$

Since

$$
\operatorname{cn}(y, k) \operatorname{dn}(y, k) \operatorname{sn}(y, k) \sim \frac{\partial}{\partial y}\left(\zeta-k^{2}-k^{2} \operatorname{sn}^{2}(y, k)+1\right),
$$

the term

$$
-\Omega(\zeta)\left(\zeta-k^{2}-k^{2} \operatorname{sn}^{2}(y, k)+1\right) \operatorname{cn}(y, k) \operatorname{dn}(y, k) \operatorname{sn}(y, k)
$$

does not contribute to $\mathcal{K}_{1}(\lambda)$, as the integral is over a number of periods of the periodic integrand. Thus, we can conclude that

$$
\mathcal{K}_{1}(\lambda)=4 \Omega^{2}(\zeta) \int_{-n K(k)}^{n K(k)}\left(\zeta-k^{2}-k^{2} \operatorname{sn}^{2}(y, k)+1\right) \mathrm{d} y .
$$

There are two parts to the Lax spectrum $\sigma_{L}$, as shown in [6, Figure 4]. As reiterated above, $\zeta \in$ $\left(-\infty, k^{2}-1\right]$ or $\zeta \in\left[2 k^{2}-1, k^{2}\right]$. This gives rise to two parts of the essential spectrum of $\partial_{y} \mathcal{L}$. The semiinfinite component of $\sigma_{L}$ gives rise to a single covering of the imaginary axis, while an additional double covering of a symmetric interval on the imaginary axis around the origin corresponds to $\zeta \in\left[2 k^{2}-1, k^{2}\right]$. Restricting ourselves to eigenfunctions with period $2 n K(k)$ results in a discrete subset of these spectral sets. Considering the above, we observe that for $\zeta \leq k^{2}-1$ the integrand in (3.5) is negative, resulting in $\mathcal{K}_{1}(\lambda) \geq 0$, with equality attained only for $\zeta=k^{2}-1$. This corresponds to the single covering of the imaginary axis having positive Krein signature. Next, when $\zeta \in\left[2 k^{2}-1, k^{2}\right]$ the integrand in (3.5) is positive so that $\mathcal{K}_{1}(\lambda) \leq 0$, with equality attained again at the endpoints only. Thus the double covering of the imaginary interval around the origin has negative Krein signature. Although informative, no orbital stability conclusion about the $\mathrm{KdV}$ cnoidal wave can be reached from these considerations since $\mathcal{K}_{1}(\lambda)$ does not have definite sign.

At this point, we invoke more tools arising from the integrability of KdV. The KdV equation is the first nontrivial equation of an infinite hierarchy of partial differential equations that have mutually commuting flows. The equations in the hierarchy dictate how the function $u$ changes under the flow of these different dynamical systems, parameterized by their respective time variable. The first three members of this hierarchy are

$$
\begin{aligned}
& u_{\tau_{0}}=u_{y} \\
& u_{\tau_{1}}=-u u_{y}-u_{y y y}+c_{10} u_{y}, \\
& u_{\tau_{2}}=\frac{5}{6} u^{2} u_{y}+\frac{10}{3} u_{y} u_{y y}+\frac{5}{3} u u_{y y y}+u_{y y y y y}+c_{21}\left(-u u_{y}-u_{y y y}\right)+c_{20} u_{y} .
\end{aligned}
$$

It is clear that $\tau_{1}=\tau$, provided $c_{10}=c$. It is common in the literature to equate all constants $c_{j k}$ to zero, but the inclusion of the lower flows with each given flow is important for our purposes, as may already be obvious from the fact that (2.2) only appears for nonzero choice of $c_{10}$. Clearly the inclusion of the lower equations does not affect the commutativity of the different flows.

Each equation in the KdV hierarchy is Hamiltonian,

$$
u_{\tau_{k}}=\partial_{y} H_{k}^{\prime}(u)
$$

where the prime denotes the variational derivative of the Hamiltonian $H$ with respect to $u$. For the $k$-th KdV equation, the Hamiltonian $H_{k}(u)$ depends on $u$ and its first $k$ derivatives with respect to $y$. For instance,

$$
H_{2}(u)=\int_{-n K(k)}^{n K(k)}\left(\frac{1}{2} u_{y y}^{2}-\frac{5}{6} u u_{y}^{2}+\frac{5}{72} u^{4}+c_{21}\left(\frac{1}{2} u_{y}^{2}-\frac{1}{6} u^{3}\right)+c_{20} \frac{1}{2} u^{2}\right) d y .
$$


Since all the flows in the KdV hierarchy commute, the cnoidal wave solution (2.3) of KdV (2.2) is a stationary (with respect to $\tau_{2}$ ) solution of the second KdV equation for a suitable choice of the constants $c_{21}$ and $c_{20}$. This fixes $c_{20}$ in terms of $c_{21}$, but it imposes no constraint on $c_{21}$, since the cnoidal wave (2.3) is a stationary solution of (2.2). Thus, $c_{21}$ is a free parameter: for any choice of $c_{21}$ the cnoidal wave solution (2.3) is a stationary periodic solution of the second KdV equation (3.8). Indeed, direct substitution of the cnoidal wave solution shows that

$$
c_{20}=c_{21}\left(8 k^{2}-4\right)-56 k^{4}+56 k^{2}-16 .
$$

We now consider the stability problem with respect to the dynamical variable $\tau_{2}$ for the cnoidal wave solution. The linear stability problem is given by

$$
v_{\tau_{2}}=\partial_{y} \mathcal{L}_{2} v
$$

with associated spectral stability problem

$$
\lambda_{2} V=\partial_{y} \mathcal{L}_{2} V, \quad v=e^{\lambda_{2} \tau_{2}} V,
$$

where

$$
\mathcal{L}_{2}=\partial_{y}^{4}+\frac{5}{3} U(y) \partial_{y}^{2}+\frac{5}{3} U^{\prime}(y) \partial_{y}+\frac{5}{3} U^{\prime \prime}(y)+\frac{5}{6} U^{2}(y)+c_{21}\left(-\partial_{y}^{2}+c-U(y)\right)-16+56 k^{2}\left(1-k^{2}\right) .
$$

The freedom in choosing $c_{21}$ gives rise to a one-parameter family of linear stability problems. The Krein signature $\mathcal{K}_{2}$ associated with the eigenvalues of the operator $\mathcal{J L}_{2}$ is the sign of

$$
\mathcal{K}_{2}:=\left\langle V, \mathcal{L}_{2} V\right\rangle=\int_{-n K(k)}^{n K(k)} V^{*} \mathcal{L}_{2} V \mathrm{~d} y
$$

The eigenfunction $V(y)$ is the same as before, due to the commuting property of the flows. This is also easily verified using a direct calculation. All eigenfunctions of $\mathcal{J} \mathcal{L}_{2}$ are obtained this way, since the eigenfunctions $V$ form a complete set in $L_{\mathrm{per}}^{2}([-n L, n L])$, see [6]. We remind the reader that this statement requires the invertibility of the Poisson operator $\partial_{y}$ of the KdV hierarchy, requiring us to fix the average of the solutions of the hierarchy, see also [9]. In other words, we are considering perturbations that do not alter the average of the cnoidal wave. Since $\mathcal{K}_{2}$ is computed for the same set of eigenfunctions as $\mathcal{K}_{1}$, we may think of $\mathcal{K}_{2}$ as being parameterized by the Lax spectral parameter $\zeta$ in the same way that $\mathcal{K}_{1}$ is.

A simple linear algebra calculation along the lines of that done in [6, Section 5] using the Lax pair of the second $\mathrm{KdV}$ equation demonstrates that

$$
\Omega_{2}^{2}(\zeta)=\Omega^{2}(\zeta)\left(4 \zeta-c_{21}+8 k^{2}-4\right)^{2} .
$$

Here $\Omega_{2}$ is the separation constant determining the exponential dependence of $\psi$ on $\tau_{2}$. Note that $\psi$ is the simultaneous solution of all Lax equations corresponding to the KdV hierarchy. Another direct calculation gives an expression for the integrand of (3.14) as

$$
\begin{gathered}
V^{*} \mathcal{L}_{2} V=64|\gamma(x)|^{4} \Omega(\zeta)\left(4 \zeta-c_{21}+8 k^{2}-4\right)\left(\zeta-k^{2}-k^{2} \operatorname{sn}^{2}(y, k)+1\right)^{3} \times \\
\left(4 k^{2} \operatorname{cn}(y, k) \operatorname{dn}(y, k) \operatorname{sn}(y, k)-\Omega(\zeta)\right) .
\end{gathered}
$$

Using the same simplifications and substitutions as above, we find the remarkable result that $\mathcal{K}_{2}(\zeta)$ is linearly related to $\mathcal{K}_{1}(\zeta)$ via the relationship

$$
\mathcal{K}_{2}(\zeta)=-\left(4 \zeta-c_{21}+8 k^{2}-4\right) \mathcal{K}_{1}(\zeta) .
$$

The factor $4 \zeta-c_{21}+8 k^{2}-4$ changes sign when $\zeta$ crosses $1-2 k^{2}+c_{21} / 4$. If we choose $c_{21}$ so that its product with $\mathcal{K}_{1}(\lambda)$ is always positive (or zero, where $\mathcal{K}_{1}(\zeta)=0$ ) for all values of $\zeta \in \sigma_{L}$, then $\mathcal{K}_{2}(\zeta)$ does not change sign. To this end we need 


$$
k^{2}-1<1-2 k^{2}+c_{21} / 4<2 k^{2}-1
$$

which is easily satisfied by choosing

$$
4\left(3 k^{2}-2\right)<c_{21}<4\left(4 k^{2}-2\right) .
$$

In conclusion, we see it is possible to choose the free parameter $c_{21}$ so that $\mathcal{K}_{2}(\zeta)$ is positive definite for any choice of the elliptic modulus $k$. Note that $\mathcal{K}_{2}(\zeta)=0$ only when $\zeta$ equals one of the three roots of the righthand side of (2.9). For these values $\lambda=2 \Omega=0$, which using (3.15) implies $\lambda_{2}=2 \Omega_{2}=0$. Thus $\mathcal{K}_{2}\left(\lambda_{2}\right) \geq 0$, with equality attained only when $\lambda_{2}=0$. This is an important observation. As shown in [6], the values of $\zeta$ for which $\Omega(\zeta)=0$ all correspond to a single eigenfunction $V \sim \operatorname{sn}(y, k) \operatorname{cn}(y, k) \operatorname{dn}(y, k) \sim \partial_{y} \operatorname{cn}^{2}(y, k)$, which is the sole generator of the Lie point symmetry group of the cnoidal wave, consisting of spatial translation. It follows that all conditions for Theorem 1 of [14] are satisfied, finishing the proof of orbital stability of the cnoidal wave solutions of the second $\mathrm{KdV}$ equation (3.8) with respect to perturbations in $L_{\text {per }}^{2}([-n K(k), n K(k)] ; \mathbb{R})$, modulo the translational symmetry. Thus we have proved the following theorem.

Theorem 3.1. Consider the solution $U(y)=12 k^{2} \mathrm{cn}^{2}(y, k)$ on the space $L_{\text {per }}^{2}([-n K(k), n K(k)] ; \mathbb{R})$ endowed with the natural inner-product, where $n \in \mathbb{N}_{0}$ is given. For a given $\epsilon>0$ sufficiently small there is a $\delta>0$ such that if $\|u-U\|<\delta$ with $\langle u\rangle=\langle U\rangle$, then

$$
\inf _{\omega \in \mathbb{R}}\left\|v\left(\tau_{2}\right)-U(\cdot+\omega)\right\|<\epsilon
$$

Now let us consider the implications of this calculation. This is where we invoke the same argument that was used in [20] to establish the orbital stability of the $N$-soliton solutions of the KdV equation. Our calculation of the Krein signature implies that the quantity (3.10), with $c_{20}$ and $c_{21}$ satisfying (3.11) and (3.16) respectively, acts as a Lyapunov functional for the cnoidal wave solutions of the KdV equation with respect to the $\tau_{2}$ dynamics. This implies that all perturbations that are not mere shifts of the cnoidal wave in $L_{\text {per }}^{2}([-n K(k), n K(k)] ; \mathbb{R})$ give rise to an increase in the value of $H_{2}(u)$. But all flows of the $\mathrm{KdV}$ hierarchy commute, and $H_{2}(u)$ is a conserved quantity with respect to the $\tau$ dynamics as well. Hence, (3.10) also allows us to establish formal stability of the cnoidal waves under the KdV dynamics. Finally, since the eigenfunctions of the linear stability problems for the $\tau$ and $\tau_{2}$ flows are identical, the Krein signature calculation necessary to invoke Theorem 1 of $[\mathbf{1 4}]$ does not need to be repeated as it remains the same. In short, orbital stability of the cnoidal waves with respect to perturbations in $L_{\mathrm{per}}^{2}([-n K(k), n K(k)] ; \mathbb{R})$ under the $\tau_{2}$ dynamics establishes orbital stability of these cnoidal waves with respect to the same class of perturbations under the $\tau$ dynamics. Thus, the cnoidal wave solutions of the KdV equation are orbitally stable with respect to subharmonic perturbations which respect the average value of the solution.

Theorem 3.2. Consider the solution $U(y)=12 k^{2} \mathrm{cn}^{2}(y, k)$ on the space $L_{\mathrm{per}}^{2}([-n K(k), n K(k)] ; \mathbb{R})$ endowed with the natural inner-product, where $n \in \mathbb{N}$ is given. For a given $\epsilon>0$ sufficiently small there is a $\delta>0$ such that if $\|v-U\|<\delta$ with $\langle u\rangle=\langle U\rangle$, then

$$
\inf _{\omega \in \mathbb{R}}\|u(t)-U(\cdot+\omega)\|<\epsilon .
$$

\section{REFERENCES}

[1] Ablowitz, M. J., and Segur, H., Solitons and the Inverse Scattering Transform. Society for Industrial and Applied Mathematics (SIAM), Philadelphia, PA, 1981.

[2] Handbook of mathematical functions, with formulas, graphs and mathematical tables. National Bureau of Standards, Washington, D.C., 1966. Edited by M. Abramowitz and I. A. Stegun.

[3] J. Angulo, J. Bona, and M. Scialom. Stability of cnoidal waves. Adv. Diff. Eq., 11:1321-1374, 2006.

[4] Benjamin, T. B., Lectures on nonlinear wave motion, in "Nonlinear Wave Motion," American Mathematical Society (1974), 3-47. 
[5] H. Bohr Almost Periodic Functions. Chelsea Publishing Company, New York, N.Y., 1947.

[6] N. Bottman and B. Deconinck. KdV cnoidal waves are linearly stable. Preprint.

[7] J. Bronski and M. Johnson. The modulational instability for a generalized KdV equation. arXiv:0809.4402v3, 2008.

[8] P. F. Byrd and M. D. Friedman, "Handbook of Elliptic Integrals for Engineers and Scientists," Springer-Verlag, New York, 1971.

[9] B. Deconinck and T. Kapitula. On the orbital (in)stability of spatially periodic stationary solutions of generalized Korteweg-de Vries equations. Submitted for publication, 2009.

[10] B. Deconinck and J.N. Kutz. Computing spectra of linear operators using Hill's method. J. Comp. Physics, 219:296-321, 2006.

[11] T. Gallay and M. Hărăguş. Orbital stability of periodic waves for the nonlinear Schrödinger equation. J. Dyn. Diff. Eqns., 19:825-865, 2007.

[12] T. Gallay and M. Hărăguş. Stability of small periodic waves for the nonlinear Schrödinger equation. J. Diff. Eq., 234:544-581, 2007.

[13] C. S. Gardner. Korteweg-de Vries equation and generalizations. IV. The Korteweg-de Vries equation as a Hamiltonian system. J. Math. Phys., 12: 1548-1551, 1971.

[14] M. Grillakis, J. Shatah, and W. Strauss. Stability theory of solitary waves in the presence of symmetry. I. Journal of Functional Analysis, 74:160-197, 1987.

[15] M. Grillakis, J. Shatah, and W. Strauss. Stability theory of solitary waves in the presence of symmetry. II. Journal of Functional Analysis, 94:308-348, 1990.

[16] M. Hărăguş and T. Kapitula. On the spectra of periodic waves for infinite-dimensional Hamiltonian systems. Physica D, 237(20):2649-2671, 2008.

[17] D. D. Holm, J. E. Marsden, T. Ratiu, and A. Weinstein. Nonlinear stability of fluids and plasma equilibria. Physics Reports, 123: 1-116, 1985.

[18] T. Kapitula. On the stability of $N$-solitons in integrable systems. Nonlinearity, 20: 879-907, 2007.

[19] P. D. Lax. Integrals of nonlinear equations of evolution and solitary waves. Comm. Pure and Appl. Math., 21: 467-490, 1968.

[20] J. H. Maddocks and R. L. Sachs. On the stability of KdV multi-solitons. Comm. Pure and Appl. Math., 46: 867-901, 1993.

[21] H. P. McKean. Stability for the Korteweg-de Vries equation. Comm. Pure and Appl. Math., 30: 347-353, 1977.

[22] J. Rademacher, B. Sandstede, and A. Scheel. Computing absolute and essential spectra using continuation. Physica D, 229(1\&2):166-183, 2007.

[23] R. L. Wiegel. A presentation of cnoidal wave theory for practical application J. Fluid Mech., 7, 273-286, 1960. 